\title{
LncRNA-miRNA-mRNA expression variation profile in the urine of calcium oxalate stone patients
}

Xiongfa Liang ${ }^{\dagger}$, Yongchang Lai ${ }^{\dagger}$, Weizhou Wu ${ }^{\dagger}$, Dong Chen, Fangling Zhong, Jian Huang, Tao Zeng, Xiaolu Duan, Yapeng Huang, Shike Zhang, Shujue Li and Wenqi Wu* (D)

\begin{abstract}
Background: To explore long-non-coding RNA (IncRNA), microRNA (miRNA) and messenger RNA (mRNA) expression profiles and their biological functions in the urine samples in calcium oxalate ( $\mathrm{CaOx}$ ) patients.

Methods: Five $\mathrm{CaOx}$ kidney stone patients were recruited in $\mathrm{CaOx}$ stone group and six healthy people were included as control group, whose midstream morning urine was collected before the patients were given any medicine on admission. After total RNA was extracted from urine, microarray of miRNA, mRNA and IncRNA were applied to explore their expression variation. Gene ontology (GO) enrichment analysis and Kyoto Encyclopedia of Genes and Genomes (KEGG) pathway analysis were performed to reveal the gene functions of the dysregulated IncRNA-associated competing endogenous RNA (ceRNA) network. Quantitative real-time PCR were performed on HK-2 cells treated with sodium oxalate $(\mathrm{NaOx})$ to further screen out the differentially expression profiles of these RNAs.

Results: A total of nine miRNAs, 883 mRNAs and 1002 IncRNAs were differentially expressed in urine of CaOx patients compared with normal population. GO analysis revealed that most of mRNAs from ceRNA network were enriched in terms of respiratory burst, regulation of mitophagy, and protein kinase regulator activity. KEGG pathway analysis of these genes related to ceRNA network highlight their critical role in pentose phosphate pathway, glyoxylate and dicarboxylate metabolism, and Janus kinase/signal transducer and activator of transcription (JAK-STAT) signaling pathway. Five miRNAs (miR-6796-3p, miR-30d-5p, miR-3192-3p, miR-518b and miR-6776-3p), four mRNAs (NT5E, CDH4, CLEC14A, CCNL1) and six IncRNAs (Inc-TIGD1L2-3, Inc-KIN-1, Inc-FAM72B-4, Inc-EVI5L-1, Inc-SERPINI1-2, Inc-MB-6) from the HK-2 cells induced by $\mathrm{NaOx}$ were consistent with the expression changes of microarray results.

Conclusion: The differential expressed miRNAs, mRNAs and IncRNAs may be associated with numerous variations of the signaling pathways or regulation of metabolism and kinase activity, providing potential biomarkers for early diagnosis of urolithiasis and new basis for further research of urolithiasis mechanism.
\end{abstract}

Keywords: Calcium oxalate, Kidney stone, Competing endogenous RNA network, Urine, LncRNA, MiRNA

\section{Background}

Urolithiasis is one of the most common diseases in department of urology with rising prevalence [1] of which kidney stones take up $6.4 \%$ prevalence in China [2]. Kidney stones lead to recurrent urinary tract infection,

\footnotetext{
* Correspondence: wwqwml@163.com

${ }^{+}$Xiongfa Liang, Yongchang Lai and Weizhou Wu contributed equally to this work.

Department of Urology, Minimally Invasive Surgery Center, The First Affiliated Hospital of Guangzhou Medical University, Guangzhou Urology Research Institute, Guangdong Key Laboratory of Urology, Kangda Road 1\#, Haizhu District, Guangzhou 510230, Guangdong, China
}

urinary tract obstruction and even renal failure which have a severe impact on the health of the patients. Accounting for $80 \%$ of all stone compositions, calcium oxalate $(\mathrm{CaOx})$ stone is the most common urinary stones that has a high recurrence rate of $40 \%$ within 5 years [3]. However, the particular mechanisms of $\mathrm{CaOx}$ stone formation remain unclear. Renal tubular epithelial cell damage caused by hyperoxaluria, oxidative stress and inflammation are involved in the process of stone formation [4].

(c) The Author(s). 2019 Open Access This article is distributed under the terms of the Creative Commons Attribution 4.0 International License (http://creativecommons.org/licenses/by/4.0/), which permits unrestricted use, distribution, and reproduction in any medium, provided you give appropriate credit to the original author(s) and the source, provide a link to the Creative Commons license, and indicate if changes were made. The Creative Commons Public Domain Dedication waiver (http://creativecommons.org/publicdomain/zero/1.0/) applies to the data made available in this article, unless otherwise stated. 
As a class of small endogenous non-protein-coding RNAs of 20-22 nucleotides, microRNAs (miRNA) lead to the target messenger RNA (mRNA) degradation or translation inhibition by combining with the 3 '-untranslated region of target mRNA [5, 6]. 31\% of the target gene expression involving in processes of cell proliferation, differentiation, development, aging, and apoptosis could be regulated by miRNA [7]. Dysregulation of miRNAs in kidney might be associated with hypercalciuria which can lead to calcium urolithiasis [8]. Long-non-coding RNA (lncRNA) is a kind of RNAs with a length greater than 200 nucleotides with many of the structural characteristics of mRNA, including a poly(A) tail, 50-capping, and a promoter structure, but not conserved open reading frames $[9,10]$. LncRNAs have been demonstrated to interact with miRNAs, imposing an additional level of post-transcriptional regulation and forming a complex regulatory network [11]. The expression profiles of lncRNAs and mRNAs in the CaOx-attached HK-2 cells, and bioinformatic analysis of lncRNA-related mRNA shown that these identified genes were involved in infectious system and signal transduction process [12].

Our previous study had investigated the miRNA and mRNA expression profile of kidney tissues in $\mathrm{CaOx}$ deposition rats and found that the biological functions of these reported miRNAs might be associated with the regulation of ion transport, inflammatory response, and response to wounding and metabolism of epithelial cells in pathogenesis of $\mathrm{CaOx}$ stone formation [13]. Although the interaction among lncRNAs, miRNAs and mRNAs in the processes of $\mathrm{CaOx}$ stones formation has been paid little attention and has not been sufficiently investigated, lncRNA LINC00339 was found to promote cell pyroptosis and immune inflammation impairment in $\mathrm{CaOx}$-treated HK-2 cells by sponging miR-22-3p to regulate NLRP3 expression [14]. Other IncRNA-miRNA and mRNA interation in renal impairment include LOC105374325-miR-34c/ $\mathrm{miR}-196 \mathrm{a} / \mathrm{b}$ and Bax/Bak, and overexpression of LINC00520 -miR-27b-3p-OSMR [15, 16]. However, although previous studies have defined the expression profiles of miRNAs and lncRNAs in kidney tissue of $\mathrm{CaOx}$ animal model or in cells, the hyperoxaluria or hypercalciuria animal model and cell model treated with $\mathrm{CaOx}$ may not really consistent with pathological alterations in clinic $\mathrm{CaOx}$ patients $[12,17]$. What's more, as it is an ethical issue to study the kidney tissue of clinic patients and the urine of urolithiasis patients may also directly reflect the associated pathological alterations of human stone disease to some extent, we collected urine of urolithiasis patients and further investigated their alterations and interaction among miRNA, mRNA and lncRNA in this study. We also used the microarray technology and bioinformatics databases to integrally analyze the miRNA, mRNA and lncRNA expression network for the urine of $\mathrm{CaOx}$ stone patients.

\section{Methods}

This research was approved by the human ethics committee of the First Affiliated Hospital of Guangzhou Medical University (Guangzhou, Guangdong, China). All patients signed the informed consent prior to the study.

\section{Patients and samples}

The samples and clinical data were collected from the Minimally Invasive Surgery Center, Department of Urology, The First Affiliated Hospital of Guangzhou Medical University (Guangzhou, China) from October 2016 to December 2016. The inclusion criteria for the patients were: 1) male patients diagnosed as calcium oxalate renal stones; 2) aged $18-65$ years. Exclusion criteria include urinary tract infection, diabetes mellitus, hypertension, dyslipidemia, dysfunction of liver and kidney, congenital dysplasia of urinary system, history of urinary tumor, renal transplantation and urinary diversion, ectopic kidney, polycystic kidney, horseshoe kidney, kidney malrotation, ureteral stenosis and other malformations. Five $\mathrm{CaOx}$ kidney stone patients were recruited in $\mathrm{CaOx}$ stone group, and six healthy people were included in control group. All participants were male, no significant difference in age, weight and height between the $\mathrm{CaOx}$ stone group and the control group was found. The detailed characteristics of study subjects are summarized in Table 1. Stone composition of $\mathrm{CaOx}$ was confirmed by infrared spectrum analysis method. Midstream morning urine was collected as specimen from all participants before patients were given any medicine on admission.

\section{Urine RNA extraction}

Total RNA was extracted from urine using mirVana ${ }^{\mathrm{Tm}}$ PARIS $^{\text {ma }}$ Kit (Ambion-1556, USA) according to the manufacturer's protocol. The purity and concentration were assessed by NanoDrop ND-2000 (Thermo Fisher, USA) and the RNA integrity was confirmed by Agilent Bioanalyzer 2100 (Agilent Technologies). The concentrate of extracted RNA was about $200-2000 \mathrm{pg} / \mu \mathrm{l}$, and the total extracted RNA over 2000 pg was used for next-step analysis. After the RNA was qualified, the samples were labeled, hybridized and eluted according to the standard procedure of microarray.

\section{Microarray analysis}

Both the samples from stone group and the samples from control group were selected for microarray analysis. LncRNA and mRNA expression profiles were analyzed by the Affymetrix ${ }^{\circ}$ GeneChip ${ }^{\circ}$ Whole Transcript Expression Arrays, and Agilent miRNA Microarrays $8 \mathrm{x} 60 \mathrm{~K}$ were applied to analyze miRNA expression profiles in the urine. Microarray analyses were performed by Oebiotech Corporation (Shanghai, China). Hierarchical clustering analysis and volcano maps were carried 
Table 1 Characteristics of the study subjects

\begin{tabular}{llll}
\hline Traits & Stone-forming group $(n=5)$ & Normal group $(n=6)$ & P Value \\
\hline Age $( \pm S D),($ years $)$ & $31.00 \pm 7.40$ & $31.67 \pm 2.81$ & 0.858 \\
Height $( \pm S D),(\mathrm{cm})$ & $166.20 \pm 3.25$ & $170.33 \pm 6.92$ & 0.969 \\
Weight $( \pm S D),(\mathrm{kg})$ & $68.20 \pm 13.06$ & $68.50 \pm 9.29$ & 0.296 \\
Mean stone burden $( \pm \mathrm{SD}),\left(\mathrm{cm}^{2}\right)$ & $2.58 \pm 1.04$ & - & - \\
Hydronephrosis, $\mathrm{n}(\%)$ & $5(100 \%)$ & - & - \\
\hline
\end{tabular}

out using the gplots and heatmap packages in the $R$ platform.

\section{Construction of IncRNA-miRNA-mRNA and functional analysis}

CeRNA network was built by the miRNAs, lncRNAs and mRNAs with significantly difference. First, the targets of differentially expressed miRNAs were predicted by miRanda and pearson correlation coefficient. MiRanda (www. microrna.org) was used to predict the miRNA responsive elements (MRE) of target genes and binding sites of detected miRNAs based on the sequences of lncRNAs and mRNAs [18]. These predicted mRNAs and lncRNAs were compared with those detected from microarray, and then the overlap RNAs were determined. To reduce false positive of paired miRNA-mRNA and miRNA-lncRNA, Pearson correlation coefficient (PCC) was used to filtering the significant paired miRNA-mRNA and miRNA-lncRNA, and the $P$ value of PCC in each pair was calculated by Fisher's asymptotic distribution [19]. Only the interactions ofnegative PCC with $P$ value $<0.05$ were included. Then shared pairs from the predicted paired miRNA-mRNA and miRNA-lncRNA by miRanda and PCC were selected for further analysis. Finally, a ceRNA network correlated with the $\mathrm{CaOx}$ stones was drawn by Cytoscape 2.8.0 (https:// cytoscape.org/) according to predicted shared pairs of miRNA-mRNA and miRNA-lncRNA. In addition, ceRNA_scores were calculated according to the following formula for construction of ceRNA regulatory network [20], and the corresponding formula is showed below.

CeRNA score $=\frac{\text { The number of MREs for the distinct shared miRNAs between the pair }}{\text { The total number MREs for all distinct miRNAs targeting the IncRNA }}$

For the sake of determining the biological functions and pathways of these target mRNAs in ceRNA network, Gene ontology (GO, http://geneontology.org/) and Kyoto Encyclopedia of Genes and Genomes (KEGG, https://www.kegg.jp/) annotations was carried out by DAVID Bioinformatics Resources.

\section{Cell culture and cell exposure}

Human Kidney Epithelial Cells, HK-2, were obtained from the American Type Culture Collection (Manassas, VA, USA) and maintained in a DMEM/F12 medium supplemented with $10 \%$ Fetal Bovine Serum and 1\% penicillin/streptomycin. Confluent cells with 70-80\% confluence were treated with $1 \mathrm{mM}$ sodium oxalate $(\mathrm{NaOx})$ for $24 \mathrm{~h}$ at $37^{\circ} \mathrm{C}$ in a 6-well plate. Total RNA extracted from these cell was used forextraction and validation.

Cell RNA extraction and quantitative real-time PCR (qRT-PCR) TRIzol reagent (Ambion, USA) was used to extract the total cell RNA according to the manufacturer's instructions and the NanoPhotometer (Biotek, USA) was used to assess the purity and concentration of the extracted RNA. The extracted RNA concentration of all sample was higher than $400 \mu \mathrm{g} / \mu \mathrm{l}$, and the $\mathrm{A} 260 / 280$ was in the range of 1.8-2.0.

For miRNA, total RNA of $2 \mu \mathrm{g}$ was used for cDNA synthesis and quantitative detection according to the protocol of the All-in-One ${ }^{\mathrm{Ts}}$ miRNA qRT-PCR Detection Kit (GeneCopoeia, Guangzhou, China). For mRNA and lncRNA, total RNA of $1 \mu \mathrm{g}$ was reverse transcribed to cDNA by using Takara reverse transcription kit (Takara, Dalian, China) and PCR was performed based on standard protocol of SYBR Premix Ex Taq (Takara, Dalian, China). The primers were obtained and synthesized by Shanghai Generay Biotech Co., Ltd. U6 was chosen as the internal controls of miRNA, while GAPDH was used as the internal controls of lncRNA and mRNA. The expression of target miRNAs, mRNAs and lncRNAs were quantified by using $2^{-\boldsymbol{\Delta} \mathbf{\Delta C q}}$ method [21]. To compare the relative expression of different groups, the control group were normalized as "1". All reactions were performed in triplicate. The

Table 2 Total differentially expressed miRNAs between stoneforming and normal group

\begin{tabular}{llll}
\hline Upregulated miRNAs & Downregulated miRNAs & Fold change & $P$ value \\
\hline hsa-miR-4723-3p & & 4.4706 & 0.0452 \\
hsa-miR-6804-3p & 3.5887 & 0.0291 \\
hsa-miR-6796-3p & & 3.2221 & 0.0486 \\
hsa-miR-6799-3p & & 3.0989 & 0.0263 \\
& hsa-miR-518b & 3.4838 & 0.0135 \\
& hsa-miR-3192-3p & 2.5749 & 0.0423 \\
& hsa-miR-30d-5p & 2.3468 & 0.0361 \\
& hsa-miR-767-3p & 1.6135 & 0.0308 \\
& hsa-miR-6776-3p & 1.5331 & 0.0231 \\
\hline
\end{tabular}


Table 3 Top 10 up- and downregulated mRNAs between stone-forming and normal group

\begin{tabular}{|c|c|c|c|c|c|}
\hline Upregulated mRNAs & Fold change & $P$ value & Downregulated mRNAs & Fold change & $P$ value \\
\hline NT5E & 11.3689 & $1.51 \mathrm{E}-06$ & RNU6-43P & 3.7423 & 0.0177 \\
\hline $\mathrm{CDH} 4$ & 8.5574 & $6.08 \mathrm{E}-05$ & BCL2L14 & 3.0199 & 0.0045 \\
\hline NBPF15 & 6.3077 & 0.0411 & UBAC1 & 2.7921 & 0.0028 \\
\hline IGL44 & 5.6031 & 4.01E-04 & GULP1 & 2.6563 & 0.0177 \\
\hline TAF1 & 4.4195 & $2.20 \mathrm{E}-04$ & CLEC14A & 2.5972 & 0.0089 \\
\hline ANGPTL3 & 4.1717 & 0.0018 & CCNL1 & 2.5964 & $6.58 \mathrm{E}-05$ \\
\hline METTL2B & 4.1713 & $3.50 \mathrm{E}-04$ & CASP4 & 2.5553 & 0.0222 \\
\hline ASCC2 & 3.9391 & 0.0497 & SLC41A3 & 2.5178 & 0.0019 \\
\hline RPS26 & 3.6909 & 0.0059 & CAMK2G & 2.4968 & 0.0198 \\
\hline NUP62CL & 3.5754 & 0.0056 & PDK4 & 2.4216 & 0.0016 \\
\hline
\end{tabular}

synthesized primer sequences of lncRNA, mRNA and lncRNA are list in Additional file 1: Table S1.

\section{Statistical analysis}

Continuous variables in Table 1 were shown in means \pm standard deviation (SD), while experement results presented in Fig. 4 were expressed as means \pm standard error (SEM). The student's t-test was applied for statistical analysis by using SPSS 13.0. $P<0.05$ was considered to be statistically significant.

\section{Result}

Alteration of miRNA, mRNA and IncRNA expression profiles The miRNA expression of the urine sample between the two groups was detected, and nine mature miRNAs which were significantly different between the two groups were identified. Compared to the control group $(\mathrm{P}<0.05)$, the deferential expressions in the urine of $\mathrm{CaOx}$ stone group revealed 1.5-fold changes, including 4 upregulated miRNAs and 5 downregulated ones (Table 2).

To further investigate potential targets of the detected miRNAs, the mRNA and lncRNA expression profiles of the same urine samples were detected using Affymetrix expression arrays. A total of 883 mRNAs and 1002 lncRNAs were found to be differentially expressed. Among the differentially expressed mRNAs, a number of 467 mRNAs were upregulated and 416 mRNAs were downregulated in the stone group compared to control group, respectively (Table 3). Among the differentially expressed lncRNAs, 790 lncRNAs were significantly increased whereas 212 lncRNAs were significantly decreased in stone group (Table 4).

MiRNA (Fig. 1a), mRNA (Fig. 1c) and lncRNA (Fig. 1e) expression patterns between $\mathrm{CaOx}$ and normal urine sample were distinguished via hierarchical clustering heatmaps. Volcano plots were performed to assess the variation and reproducibility of miRNA (Fig. 1b), mRNA (Fig. 1d) and lncRNA (Fig. 1f) expression in urine between $\mathrm{CaOx}$ and normal group.

In line with our results, 131 mRNAs such as $5^{\prime}$ nucleotidase, ecto (NT5E), B-cell lymphoma 2 like 14 (BCL2L14), ubiquitin associated domain containing 1 (UBAC1), lamin A/C (LMNA), and chemokines chemokine ligand 3 (CCL3) and one miRNAs, miR-30d-5p, which were previously shown to be up-regulated or down-regulated in $\mathrm{CaOx}$ model were also found in this study (Fig. 2) [13]. Although network analysis of the above signaling pathways are not constructed, the Venn diagram were constructed and those identified mRNAs

Table 4 Top 10 up- and downregulated IncRNAs between stone-forming and normal group

\begin{tabular}{llllll}
\hline Upregulated mRNAs & Fold change & P value & Downregulated miRNAs & Fold change & P value \\
\hline Inc-STX11-4 & 9.2496 & 0.0435 & Inc-WHSC1L1-11 & 2.9871 & 0.0091 \\
Inc-ALKBH4-8 & 7.7525 & 0.0363 & Inc-FAM72B-4 & 2.5733 & 0.0013 \\
Inc-KIF24-2 & 6.8555 & 0.0342 & RNY4 & 2.5414 & 0.0230 \\
Inc-CHCHD7-9 & 6.5116 & 0.0454 & RP11-717A5.2 & 2.4464 & 0.0013 \\
Inc-BAGE4-3 & 6.0793 & 0.0372 & Inc-EVI5L-1 & 2.2651 & 6.11 E-04 \\
Inc-TIGD1L2-3 & 5.6854 & 0.0309 & SNAR-C4 & 2.2332 & 0.0062 \\
Inc-OPRD1-5 & 5.5862 & 0.0184 & Inc-GPR31-3 & 2.2059 & 0.0212 \\
Inc-KIN-1 & 5.4314 & 0.0157 & Inc-SERPINI1-2 & 2.1009 & 0.0057 \\
Inc-VPS45-7 & 5.3865 & 0.0372 & C12orf36 & 2.0226 & 0.0023 \\
Inc-C12orf53-1 & 5.2731 & 0.0322 & Inc-MB-6 & 2.0219 \\
\hline
\end{tabular}


A Hierarchical clustering of miRNAs

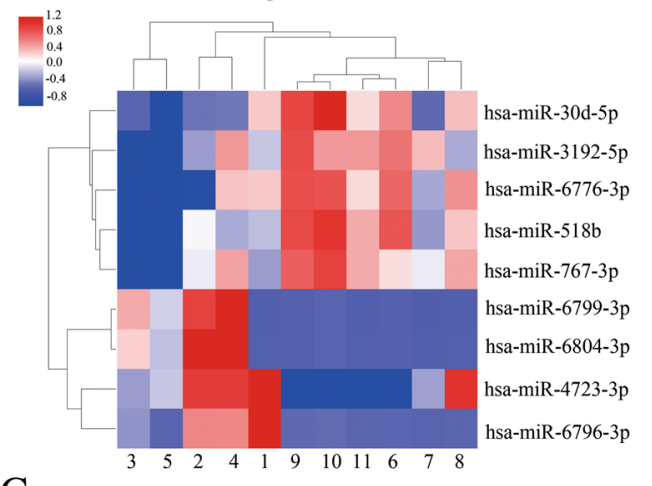

C Hierarchical clustering of mRNAs

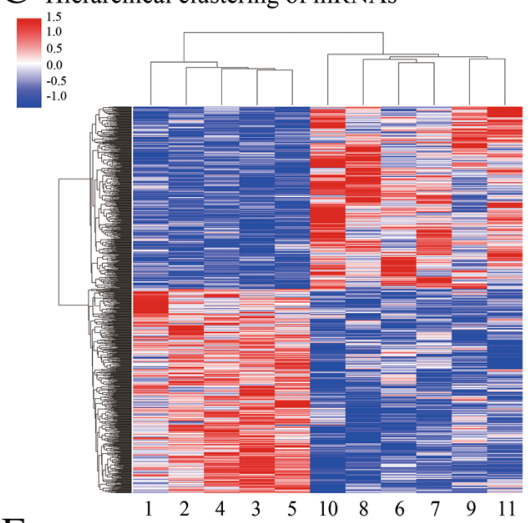

E Hierarchical clustering of IncRNAs

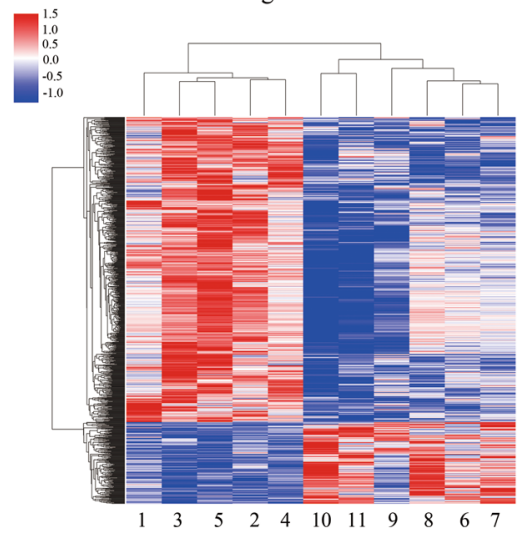

B Volcano plots of miRNAs

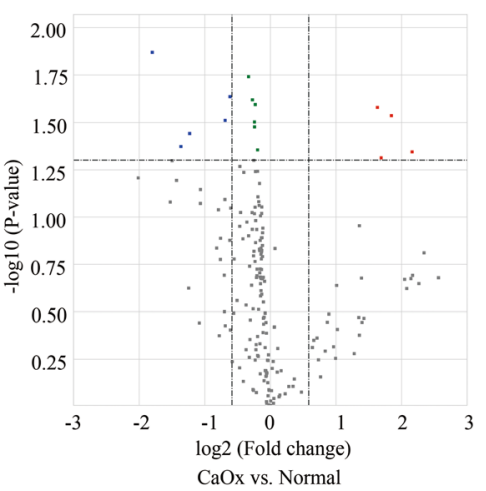

D Volcano plots of mRNAs

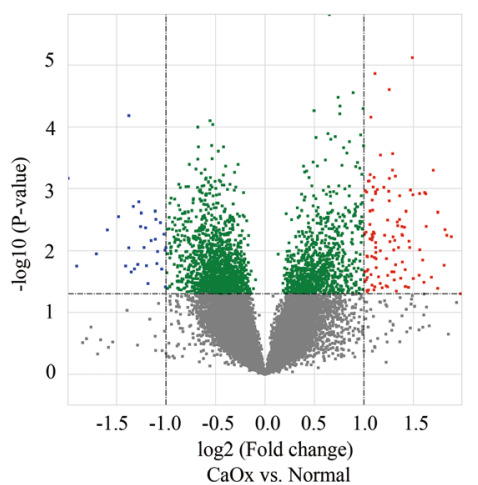

F Volcano plots of IncRNAs

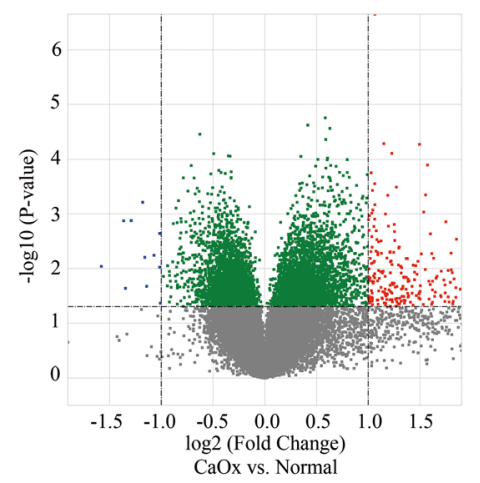

Fig. 1 Hierarchical clustering and volcano plots of dysregulated miRNA, mRNA and IncRNA. Hierarchical clustering shows all dysregulated miRNAs (a), mRNAs (c) and IncRNAs (e) in the urine sample of CaOx group (sample 1-5) and normal group (sample 6-11). Red represents high relative expression and blue represents low relative expression. Volcano plots were performed to assess the variation and reproducibility of miRNAs (b), mRNAs (d) and IncRNAs (f) expression between $\mathrm{CaOx}$ and normal urine sample. Red spots indicate upregulated genes, while blue spots represent downregulated genes

and miRNA may provide a following reference for the corresponding biomarker research.

\section{Construction and functional analysis of ceRNA network in urine sample}

In order to figure out the relationships among these differentially expressed RNAs, the ceRNA network among differentially expressed miRNA, mRNA and IncRNA in urine were constructed. Regulatory relationship between differentially expressed miRNA and the targeted differentially expressed mRNA or IncRNA (miRNA-mRNA or miRNA-lncRNA) were predicted by miRanda and PCC. Then the predicted shared pairs of miRNA-mRNA and miRNA-lncRNA were selected for construction of ceRNA regulatory network. A ceRNA network correlated with the $\mathrm{CaOx}$ stones was drawn by Cytoscape after integrating the miRNA-mRNA interactions into lncRNA-miRNA interactions (Fig. 3). The ceRNA network was composed of nine 
A Differential miRNA in different kind of samples

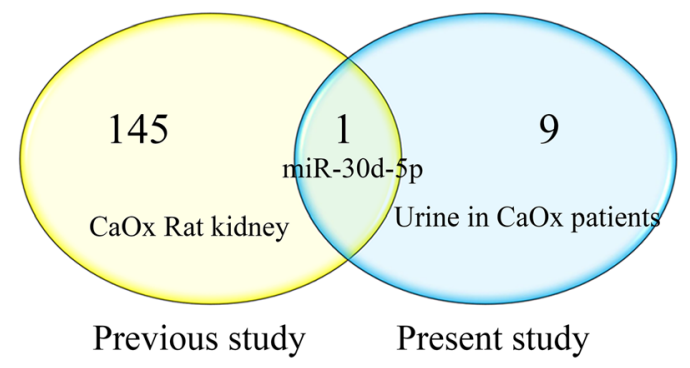

B Differential mRNA in different kind of samples

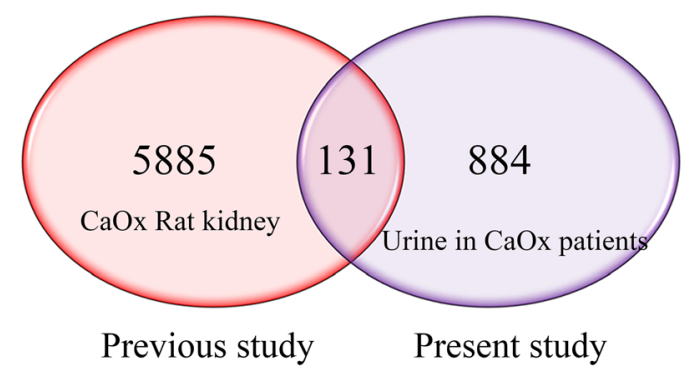

Fig. 2 Venn diagram of dysregulated mRNA and miRNA expression profile detected in two studies. a One miRNA was found to be significant both in our previous studies in the $\mathrm{CaOx}$ rat model and in this study of urine from CaOx patient; (b) 131 mRNAs were found to be significant both in our previous studies and in our present study. $\mathrm{CaOx}$, calcium oxalate

miRNA nodes, 141 mRNA nodes and 65 lncRNA nodes. In the ceRNA network, hsa-miR-6776-3p targeted gene $\mathrm{CDH} 4$, a gene encoding retinal type I cadherin, which can activate c-Jun $\mathrm{NH}(2)$-terminal kinase (JNK) signal pathways in osteosarcoma cells, leading to production of reactive oxygen species (ROS) [22, 23]. Several lncRNAs such as lnc-ABCA10-1:1, lnc-CANX-2:1, lnc-RDH8-4:1 and lnc-AC006156.1-12:1 were shown to share the common miRNA, hsa-miR-6776-3p, with CDH4, regulating production of ROS.

Then, the differentially expressed mRNAs reflected in the network were utilized to reveal the gene functions of the dysregulated lncRNA-associated ceRNA network via GO and KEGG pathway analysis. In GO analysis, gene function enrichment in biological process, molecular function and cellular component were analyzed $(P<0.05)$ and the top five enriched GO terms of the three aspects are listed in Table 5. GO analysis of biological process revealed that differentially expressed mRNAs were primarily enriched in positive regulation of respiratory burst, cardiac ventricle morphogenesis and positive regulation of mitophagy. The cellular component results indicated that differentially expressed mRNAs were mainly enriched in MPP7-DLG1-LIN7 complex, GATOR2 complex and nuclear exosome (RNase complex). The molecular function results showed that differentially expressed mRNAs were mainly enriched in protein kinase regulator activity, chloride ion binding and transaminase activity. In KEGG pathway analysis, the top five enriched pathways included pentose phosphate pathway, pentose and glucuronate interconversions, glyoxylate and dicarboxylate metabolism, fructose and mannose metabolism and Janus kinase/signal transducer and activator of transcription (JAK-STAT) signaling pathways (Table 6).

\section{Validation of differentially expressed miRNAs, mRNAs and IncRNAs in HK-2 cells treated with $\mathrm{NaOx}$}

To further screen out the differentially expression profiles of miRNAs, mRNA, and lncRNA, qRT-PCR were performed on HK-2 cells incubated by $\mathrm{NaOx}$. All differentially expressed miRNAs, ten mRNAs and 10 lncRNAs with high fold change filtered from microarray were selected. Relative expression levels of the selected miRNA, mRNA and lncRNA were depicted in Fig. 4 (A-F). The genes that are consistent with the expression changes of microarray will be more convinced and more likely to be the biomarkers for kidney stones. The qRT-PCR results showed that hsa-miR-6796-3p, hsa-miR-30d-5p, hsamiR-3192-3p, hsa-miR-518b and hsa-miR-6776-3p were found to be accord with the expression changes of microarray. For mRNAs, NT5E, CDH4, CLEC14A and CCNL1 were validated to be consistent with expression changes of microarray results. For IncRNAs, the expression of lnc-TIGD1L2-3 and lnc-KIN-1 were significantly increased, while lnc-FAM72B-4, lnc-EVI5L-1, lnc-SERPINI12 and lnc-MB- 6 were shown to be downregulated in HK-2 cells incubated by $\mathrm{NaOx}$. The variation tendency of o ther miRNAs, mRNAs and lncRNAs were not matched with the variations in $\mathrm{NaOx}$ induced $\mathrm{HK}-2$ cells model, which may have lower possiblity for prediction of $\mathrm{CaOx}$ kidney stones formation.

\section{Discussion}

Nephrolithiasis is a multifactorial disease with a rising prevalence. Numerous hypotheses have been investigated in previous research to clarify the mechanisms of $\mathrm{CaOx}$ stone formation [24-27]. Better understanding of the precise mechanisms of $\mathrm{CaOx}$ stones formation related to functional RNAs is critical for exploring potential new strategies for early diagnosis and therapy. In this study, we systematically analyzed the IncRNA-involved regulatory networks based on microarray data of urine from $\mathrm{CaOx}$ patients. This is our first attempt to explore a comprehensive molecular event leading to the pathogenesis of $\mathrm{CaOx}$ stones formation and discovered functional RNAs regulatory networks in the stones formation in urine sample from clinical $\mathrm{CaOx}$ patients. 


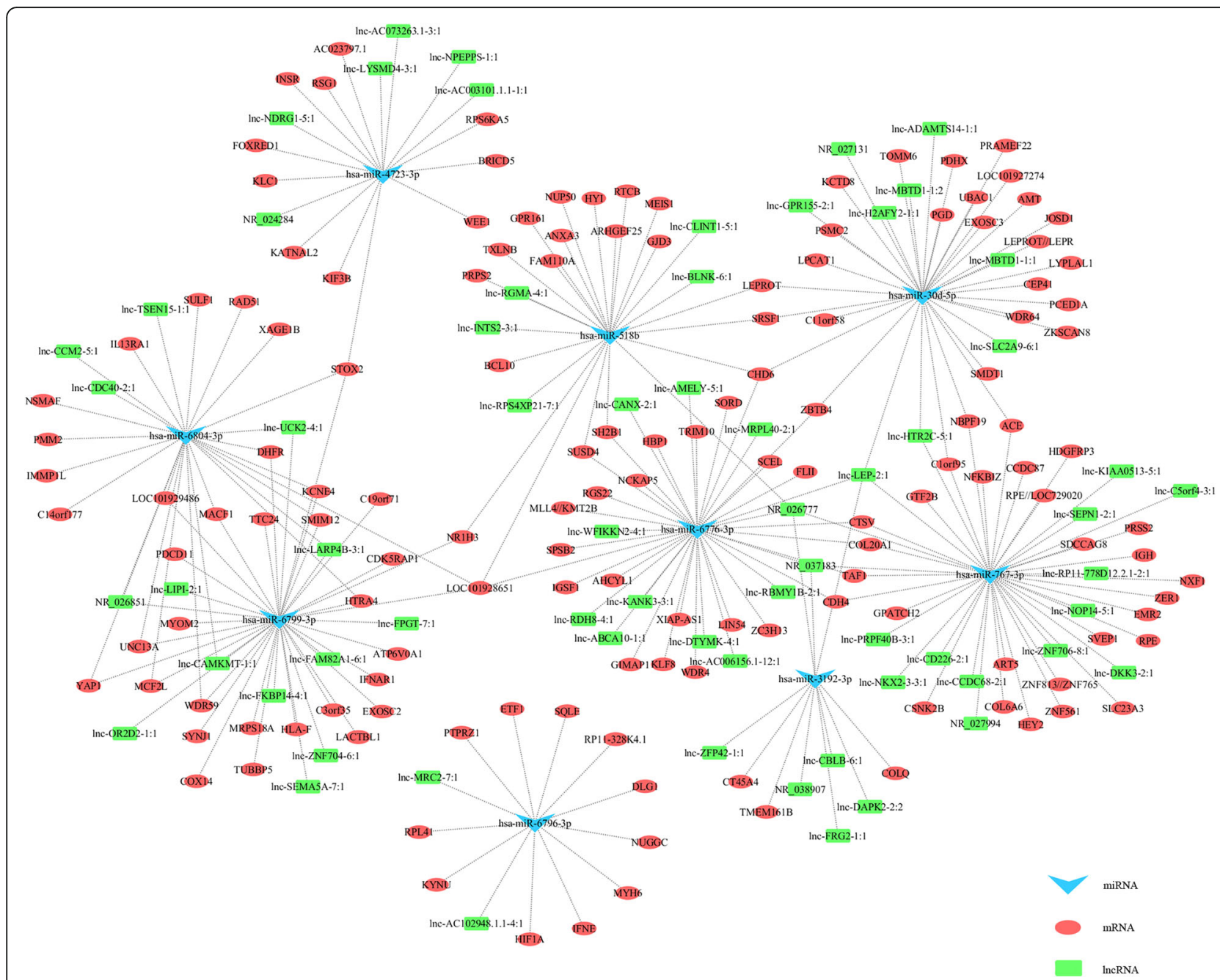

Fig. 3 CeRNA network responded to the miRNA, mRNA and IncRNA variations in urine of CaOx stone patients. There were nine miRNA nodes, 141 mRNA nodes and 65 IncRNA nodes in this network. Blue triangles represent miRNAs, green squares represent IncRNAs, and red circles represent coding genesz

Metabolite is highly correlated with $\mathrm{CaOx}$ kidney stone and the urine sample is one of the most direct and convenient body fluid for test. In the present study, we found nine miRNAs, 883 mRNAs and 1002 lncRNAs differentially expressed in urine between $\mathrm{CaOx}$ patients and the controls. In accordance with our previous study on integrative analysis miRNA and mRNA expression profiles, some previously identified differentially expressed mRNAs and miRNAs were also detected in this study [13]. We also compared the results from our previous study in $\mathrm{CaOx}$ model and the present study in urine samples, and 131 mRNAs and one miRNAs, miR-30d-5p, were also found to be consistent in this study. This RNAs changing profiles consistent in different sources of samples may be taken as biomarkers for early diagnosis or prognosis of clinic $\mathrm{CaOx}$ stone patients. Most of these detected RNAs had been found to be associated with the stones formation. LMNA expression was increased in renal tubular cells adhered with calcium oxalate monohydrate (COM), and expression of LMNA in renal tubular cells is important for tissue repair, cell proliferation, and COM crystal adhesion [28]. Inflammatory response and phagocytic mechanisms of macrophages exposed to COM crystal were also verified, and an inflammatory cascade including CCL3 was released by macrophages [29]. Increase of miR-30d-5p resulted in reduction of autophagy [30], and autophagy has been proved to be significant for the regulation of oxidative stress-induced renal tubular injury in $\mathrm{CaOx}$ stones formation [31].

As the difference was existed among the urine of renal stone patients, animal model and cell models, it is true that some of the miRNAs, mRNAs and lncRNAs may not match in $\mathrm{NaOx}$ treated HK-2 cells compared to the urine sample. In this study, the expression of several miRNAs, mRNAs and lncRNAs were confirmed in renal cell injury model by $\mathrm{NaOx}$ via qRT-PCR and the 
Table 5 The top 5 enriched Gene Ontology analysis of the ceRNA network

\begin{tabular}{|c|c|c|c|c|c|}
\hline GO ID & Category & GO term & Target genes & Fold Enrichment & $P$ Value \\
\hline GO:0060267 & Biological process & Positive regulation of respiratory burst & INSR;IGHA2 & 43.56349 & 0.00160 \\
\hline GO:0003208 & Biological process & Cardiac ventricle morphogenesis & HEY2;HIF1A & 32.66865 & 0.00255 \\
\hline GO:1903599 & Biological process & Positive regulation of mitophagy & HIF1A & 25.93386 & 0.04539 \\
\hline GO:0097084 & Biological process & Vascular smooth muscle cell development & HEY2 & 25.93386 & 0.04539 \\
\hline GO:0051000 & Biological process & Positive regulation of nitric-oxide synthase activity & DHFR;HIF1A & 25.93386 & 0.04539 \\
\hline GO:0097025 & Cellular component & MPP7-DLG1-LIN7 complex & DLG1 & 25.99559 & 0.04525 \\
\hline GO:0061700 & Cellular component & GATOR2 complex & WDR59 & 25.99559 & 0.04525 \\
\hline GO:0000176 & Cellular component & Nuclear exosome (RNase complex) & EXOSC3;EXOSC2 & 20.13561 & 0.00577 \\
\hline GO:0000178 & Cellular component & Exosome (RNase complex) & EXOSC3;EXOSC2 & 18.69629 & 0.00656 \\
\hline GO:0032982 & Cellular component & Myosin filament & MYOM2;MYH6 & 16.35741 & 0.00828 \\
\hline GO:0019887 & Molecular function & Protein kinase regulator activity & CSNK2B;CDK5RAP1 & 32.84127 & 0.00252 \\
\hline GO:0031404 & Molecular function & Chloride ion binding & ACE & 26.07087 & 0.04515 \\
\hline GO:0008483 & Molecular function & Transaminase activity & AMT & 26.07087 & 0.04515 \\
\hline GO:0034711 & Molecular function & Inhibin binding & IGSF1 & 26.07087 & 0.04515 \\
\hline GO:0031434 & Molecular function & Mitogen-activated protein kinase kinase binding & ACE;DLG1 & 17.50794 & 0.00736 \\
\hline
\end{tabular}

outcomes were basically consistent with the microarray data. The genes, such as miR-30d-5p, CDH4 were in accord with the microarray variation, which is associated with pathological alteration in stone formation, and could serve as potential biomarker for diagnosis or therapeutic target of renal stone. The inconsistent results may be related to the differences of sample sources, as they were confirmed by cell models induced by $\mathrm{NaOx}$ instead of by urine samples from clinical patients.

To further explore the role of these differentially expressed RNAs in the formation of $\mathrm{CaOx}$ stones, ceRNA network was constructed and GO and KEGG pathway analysis were carried out. The positive regulation of respiratory burst, mitophagy and nitric-oxide synthase activity, which are related to the formation of $\mathrm{CaOx}$ stone were revealed. Strong up-regulation of respiratory burst involving nicotinamide adenine dinucleotide phosphate (NADPH) oxidase system has shown to be the key cause of oxalate- or $\mathrm{CaOx}$ - induced oxidative stress-mediated renal tubular cell injury, leading to the formation of stones [32-35]. In cellular component fold enrichment of GO analysis, MPP7-DLG1-LIN7 complex was the most enriched, which is a heterotrimeric protein complex formed by the union of membrane palmitoylated protein 7 (MPP7), discs large 1 (DLG) and protein lin-7 homolog (LIN7), regulating the stability of cell junction (http://www.geneontology.org). Cell junction always play important role in regulating reabsorption of calcium and maintaining polarity of cells [36]. COM crystals would give rise to disruption of renal tubular epithelial cell tight junction, which triggered epithelial cell injury, inflammation and ultimately resulted in cell apoptosis or death by activating serine/threonine kinase (Akt), Protein Kinase B, signaling pathways or p38 mitogen-activated protein kinase (MAPK) pathways [37]. In GO analysis of molecular function, the mostly highly enriched in molecular function are protein kinase regulator activity, chloride ion binding and MAPK kinase binding Growing evidences showed that protein phosphorylation played a vital role in the development of $\mathrm{CaOx}$ kidney stones. Increased phosphorylated Akt (p-Akt) expression contributed to the epithelial-mesenchymal transition (EMT) of renal epithelial cell treated by COM crystal [38]. In another study, phosphorylation of MAPK was involved in COM crystal-induced damage, causing tight junction disruption [37].

According to the enriched KEGG pathway analysis, most of genes reflected in the ceRNA network were related to pentose phosphate pathway, pentose and glucuronate interconversions, glyoxylate and dicarboxylate metabolism, and

Table 6 The top 5 enriched pathways analysis of the ceRNA network

\begin{tabular}{lllrr}
\hline KEGG ID & KEGG term & Target genes & Fold Enrichment & $P$ Value \\
\hline path:hsa00030 & Pentose phosphate pathway & RPEL1;RPE;PGD;PRPS2 & 17.6731 & 0.00014 \\
path:hsa00040 & Pentose and glucuronate interconversions & RPEL1;RPE;SORD & 10.4727 & 0.00398 \\
path:hsa00630 & Glyoxylate and dicarboxylate metabolism & AMT;HYI & 8.8265 & 0.02526 \\
path:hsa00051 & Fructose and mannose metabolism & SORD;PMM2 & 7.7188 & 0.03189 \\
path:hsa04630 & JAK-STAT signaling pathway & IL13RA1;LEPR;IFNE;IFNAR1 & 3.2251 & 0.04288 \\
\hline
\end{tabular}


A qPCR confirmed miRNAs accord with microarray

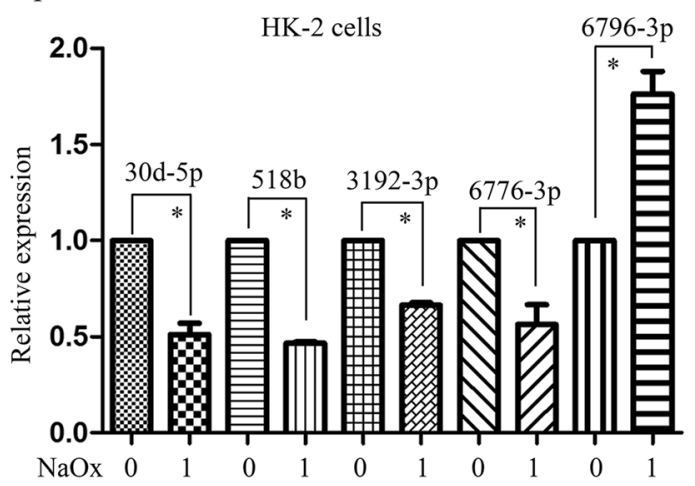

$\mathrm{C}$ qPCR confirmed mRNAs accord with microarray

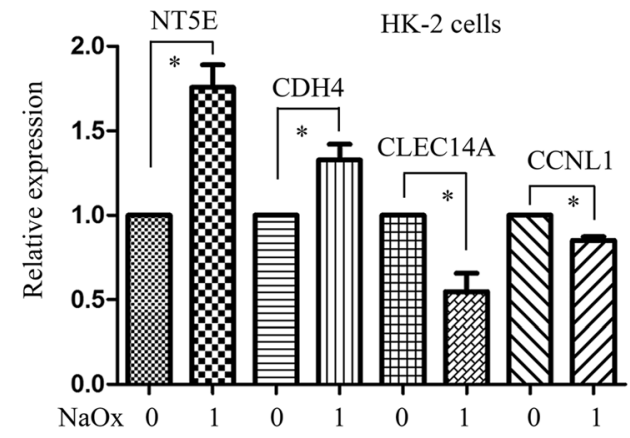

E qPCR confirmed lncRNAa accord with microarray

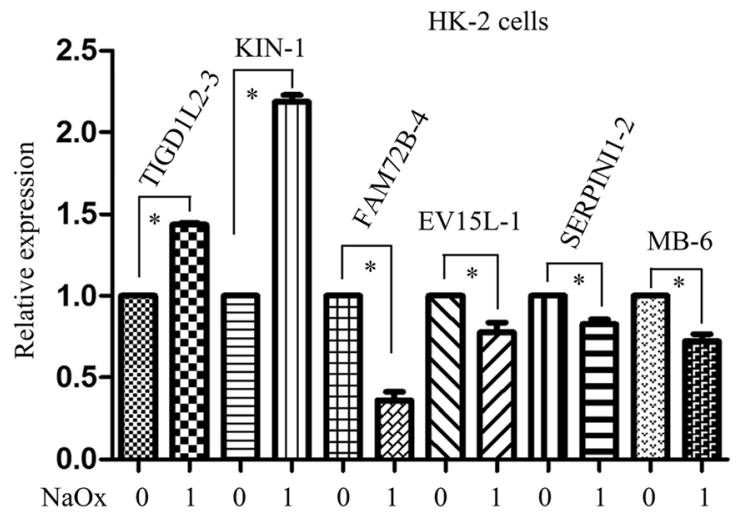

B qPCR confirmed miRNAs unsatisfied with microarray

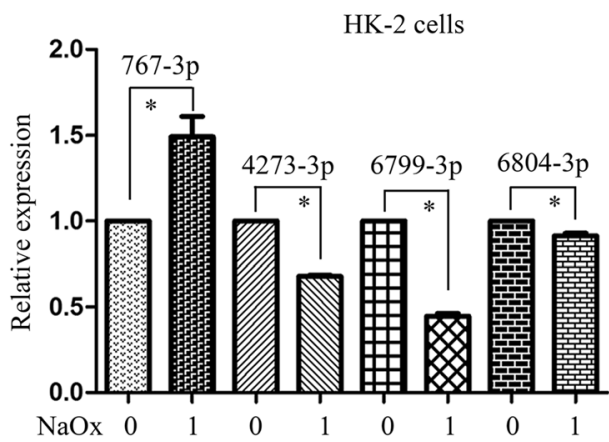

D qPCR confirmed mRNAs unsatisfied with microarray

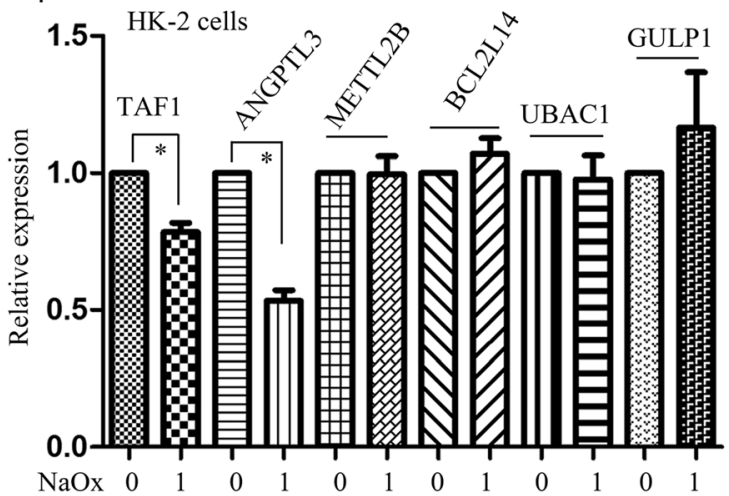

$\mathrm{F}$ qPCR confirmed lncRNAs unsatisfied with microarray

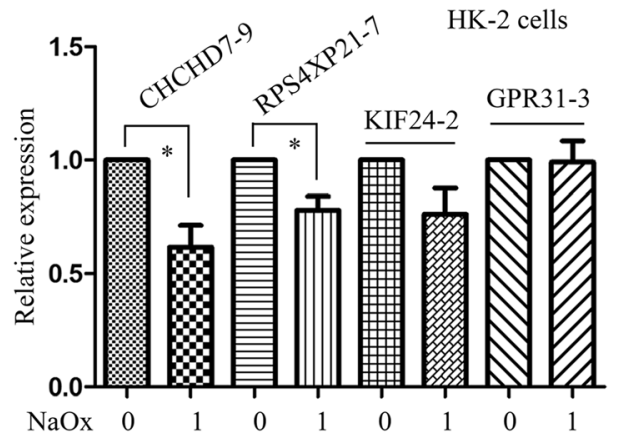

Fig. 4 qRT-PCR was performed to confirm the expression of nine miRNAs, 10 selected mRNAs and 10 selected IncRNAs in HK-2 cells treated by $\mathrm{NaOx}$. a miRNAs accord with microarray results confirmed by qRT-PCR; (b) miRNAs unsatisfied with microarray results confirmed by qRT-PCR; (c) mRNAs accord with microarray confirmed by qRT-PCR; (d) mRNAs unsatisfied with microarray results confirmed by qRT-PCR; (e) IncRNAs accord with microarray results confirmed by qRT-PCR and (f) IncRNAs unsatisfied with microarray results confirmed by qRT-PCR. Results are presented as mean \pm SEM. * represents $P<0.05$ in comparison with the corresponding group. $n=3 . n=1$ represent an independent cell culture

JAK-STAT signaling pathway. The main function of pentose phosphate pathway is to generate NADPH, which serves as a coenzyme in a wide range of oxidation-reduction reactions. NADPH is essential for maintaining levels of reduced glutathione (GSH), which is a significant contributor to endogenous antioxidant processes [39-42]. ROS could promote the formation of kidney $\mathrm{CaOx}$ stone with signaling molecule changes, renal tubular cell injury and inflammation, and treatment with antioxidants significantly reduced the crystal deposition [33, 34].

Hyperoxaluria caused by excessive accumulation of glyoxylate was another factor participated in the process of $\mathrm{CaOx}$ stone formation [43]. JAK/STAT signaling has emerged as key roles in EMT [44]. While EMT induced 
by $\mathrm{COM}$ or oxalate was involved in renal fibrosis, contributing to $\mathrm{CaOx}$ stone formation $[45,46]$. All functional analysis of this ceRNA network further indicated that the lncRNAs with ceRNA potential may play key roles in $\mathrm{CaOx}$ stone formation.

In the ceRNA network, hsa-miR-6776-3p targeted gene $\mathrm{CDH} 4$, and both hsa-miR-6776-3p and CDH4 were listed as top dysregulated RNA. In the process of urolithiasis, $\mathrm{CaOx}$ activated NADPH oxidase, producing ROS, via JNK pathway, which is activated by overexpression of $\mathrm{CDH} 4$ in osteosarcoma cells. Our qRT-PCR validation results of hsa-miR-6776-3p and CDH4 in $\mathrm{NaOx}$ treated HK-2 cells were also in accordance with microarray analysis. The ceRNA network showed that several IncRNAs such as lnc-ABCA10-1:1, lnc-CANX-2:1, lnc-RDH8-4:1 and lnc-AC006156.1-12:1 regulating the interaction between hsa-miR-6776-3p and CDH4. Among these lncRNAs, lnc-RDH8-4:1 has the highest fold change (3.0978) of deferential expressions, which may have the largest possibility to regulate hsa-miR-6776-3p-CDH4 interation and its downstream JNK pathway, finally with the production of ROS. These results indicated that lnc-RDH8-4:1, hsa-miR-6776-3p and CDH4 might serve as potential biomarker for diagnosis of renal stone by test of urine from $\mathrm{CaOx}$ patients.

In summary, instead of kidney tissue from animal model or cells, the urine from stone patients was analyzed by microarray to determine miRNAs, mRNAs and lncRNAs expression profiles in this study, which may directly reflect the role of these RNA in the formation of $\mathrm{CaOx}$ stones, providing new biomarkers for early diagnosis and prognosis of urolithiasis. However, as there is a fundamental difference between urine and kidney, whether the miRNAs, mRNAs and lncRNAs observed in urine are consistent with the natural pathogenesis of $\mathrm{CaOx}$ stone in kidney are still under investigated. In addition, HK-2 cells treated with $\mathrm{NaOx}$ were used to validate the differentially expression profiles. The verification results on the cells did not fully match the results of the array, which may be related to the different sample sources. In fact, the urine samples from clinical patients may also vary in different patients and the large sample multi-centre study of this kind of urine samples is further needed in future. Moreover, whether the altered mRNAs could be translated into the similar proteins need further exploration.

\section{Conclusion}

Taken together, the different expression of miRNAs, mRNAs and lncRNAs in the urine between $\mathrm{CaOx}$ stone patients and normal population were well defined in this study, and GO and KEGG analysis were used to reveal the gene functions of the dysregulated lncRNA-associated
ceRNA network, which included the respiratory burst, regulation of mitophagy, protein kinase regulator activity, pentose phosphate pathway, glyoxylate and dicarboxylate metabolism, and JAK-STAT signaling pathway. This study may provide new diagnosis biomarkers or treament target for renal $\mathrm{CaOx}$ stone by test of urine from patients and new direction basis for further research on urolithiasis mechanism.

\section{Additional file}

Additional file 1: Table S1. qRT-PCR primer sequences. (DOCX 19 kb)

\section{Abbreviations}

BCL2L14: B-cell lymphoma 2 like 14; CaOx: Calcium oxalate; CCL3: Chemokines chemokine ligand 3; ceRNA: competing endogenous RNA; COM: Calcium oxalate monohydrate; EMT: Epithelial-mesenchymal transition; GO: Gene ontology; GSH: Glutathione; JAK-STAT: Janus kinase/ signal transducer and activator of transcription; JNK: C-Jun NH(2)-terminal kinase; KEGG: Kyoto Encyclopedia of Genes and Genomes; LMNA: Lamin A/C; IncRNA: long-non-coding RNA; MAPK: Mitogen-activated protein kinase; MiRNA: MicroRNA; MREs: MiRNA response elements; mRNA: Messenger RNA; NADPH: Nicotinamide adenine dinucleotide phosphate; NaOx: Sodium oxalate; NT5E: 5' nucleotidase, ecto; PCC: Pearson correlation coefficient; qRTPCR: Quantitative real-time PCR; ROS: Reactive oxygen species; SD: Standard deviation; SEM: Standard error of mean; UBAC1: Ubiquitin associated domain containing 1

\section{Acknowledgements}

Not applicable.

\section{Funding}

This work was financially supported in part by research grants from Science and Technology Project of Guangzhou (201604020001), Guangzhou Science Technology and Innovation Commission (201607010162) and National Natural Science Foundation of China (81570633). The fund was mainly used for microarray analysis and examinations of subjects in study such as CT scan and chemical composition of urinary stones.

\section{Availability of data and materials}

The datasets used and/or analysed during the current study are available from the corresponding author on reasonable request.

\section{Author's contributions}

WQW and XD designed this study. $\mathrm{XL}$ and $\mathrm{YL}$ drafted the manuscript. $\mathrm{XL}, \mathrm{WZW}$, $D C$ and $F Z$ conducted the inclusion criteria for participant and urine sample collection and performed the experiment. TZ, YH, JH and SZ critically discussed and revised the manuscript. $X \mathrm{~L}, \mathrm{YL}$ and $\mathrm{SL}$ analyzed the data and revised the manuscript. All authors had read and approved the final manuscript.

\section{Ethics approval and consent to participate}

This research was approved by the human ethics committee of the First Affiliated Hospital of Guangzhou Medical University (Guangzhou, Guangdong, China). All patients signed the informed consent prior to the study.

Consent for publication Not applicable.

\section{Competing interests}

The authors declare that they have no competing interests.

\section{Publisher's Note}

Springer Nature remains neutral with regard to jurisdictional claims in published maps and institutional affiliations. 


\section{Received: 28 October 2018 Accepted: 12 April 2019}

\section{Published online: 29 April 2019}

\section{References}

1. Coe FL, Evan A, Worcester E. Kidney stone disease. J Clin Investig. 2005; 115(10):2598.

2. Zeng G, Mai Z, Xia S, Wang Z, Zhang K, Wang L, et al. Prevalence of kidney stones in China: an ultrasonography based cross; ectional study. BJU Int. 2017;120(1):109-16.

3. Worcester EM, Coe FL. Clinical practice. Calcium kidney stones. N Engl J Med. 2010;363(10):954-63.

4. Khan SR. Crystal-induced inflammation of the kidneys: results from human studies, animal models, and tissue-culture studies. Clin Exp Nephrol. 2004; 8(2):75-88.

5. Ambros V. microRNAs : tiny regulators with great potential. Cell. 2001;107(7): 823-6.

6. Ambros $V$. The functions of animal microRNAs. Nature. 2004;431(7006):350-5.

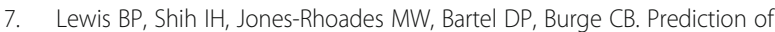
mammalian MicroRNA targets. Cell. 2003;115(7):787-98.

8. Lu Y, Qin B, Hu H, et al. Integrative microRNA-gene expression network analysis in genetic hypercalciuric stone-forming rat kidney. PeerJ. 2016;4(7):e1884

9. Chen G, Wang Z, Wang D, Qiu C, Liu M, Chen X, et al. LncRNADisease: a database for long-non-coding RNA-associated diseases. Nucleic Acids Res. 2013:41(Database issue):D983-6.

10. Gutschner T, Diederichs $S$. The hallmarks of cancer: a long non-coding RNA point of view. RNA Biol. 2012;9(6):703-19.

11. Juan L, Wang G, Radovich M, Schneider BP, Clare SE, Wang Y, et al. Potential roles of microRNAs in regulating long intergenic noncoding RNAs. BMC Med Genet. 2013;6(S1):S7.

12. Wang Z, Zhang JW, Zhang Y, Zhang SP, Hu QY, Liang H. Analyses of long non-coding RNA and mRNA profiling using RNA sequencing in calcium oxalate monohydrate-stimulated renal tubular epithelial cells. Urolithiasis. 2018. https://doi.org/10.1007/s00240-018-1065-7.

13. Lan C, Chen D, Liang $X$, Huang J, Zeng T, Duan $X$, et al. Integrative analysis of miRNA and mRNA expression profiles in calcium oxalate nephrolithiasis rat model. Biomed Res Int. 2017;2017(7):1-9.

14. Song Z, Zhang $Y$, Gong B, Xu H, Hao Z, Liang C. Long noncoding RNA LINC00339 promotes renal tubular epithelial pyroptosis by regulating the miR-22-3p/NLRP3 axis in calcium oxalate-induced kidney stone. J Cell Biochem. 2019. https://doi.org/10.1002/jcb.28330 [Epub ahead of print].

15. Hu S, Han R, Shi J, Zhu X, Qin W, Zeng C, et al. The long noncoding RNA LOC105374325 causes podocyte injury in individuals with focal segmental glomerulosclerosis[J]. J Biol Chem. 2018;293(52):20227-39.

16. Tian X, Ji Y, Liang Y, Zhang J, Guan L, Wang C. LINC00520 targeting miR$27 b-3 p$ regulates OSMR expression level to promote acute kidney injury development through the PI3K/AKT signaling pathway. J Cell Physiol. 2019. https://doi.org/10.1002/jcp.28118 [Epub ahead of print]

17. Cao Y, Gao X, Yang Y, Zhi Ye EW, Dong Z. Changing expression profiles of long non-coding RNAs, mRNAs and circular RNAs in ethylene glycolinduced kidney calculi rats[J]. BMC Genomics. 2018. https://doi.org/10.1186/ s12864-018-5052-8

18. Salmena L, Poliseno L, Tay $Y$, Kats $L$, Pandolfi PP. A ceRNA hypothesis: the Rosetta stone of a hidden RNA language? Cell. 2011;146(3):353-8.

19. Guttman M, Amit I, Garber M, French C, Lin MF, Feldser D, et al. Chromatin signature reveals over a thousand highly conserved large non-coding RNAs in mammals. Nature. 2009;458(7235):223-7.

20. Shaoli D, Suman G, Rituparno S, Jayprokas C, Sandro B. LnCeDB: database of human long noncoding RNA acting as competing endogenous RNA. PLoS One. 2014;9(6):e98965.

21. Livak KJ, Schmittgen TD. Analysis of relative gene expression data using real-time quantitative $P C R$ and the 2(-Delta Delta $C(T))$ method. Methods. 2001;25(4):402-8.

22. Tang Q, Lu J, Zou C, Shao Y, Chen Y, Narala S, et al. CDH4 is a novel determinant of osteosarcoma tumorigenesis and metastasis. Oncogene. 2018:37(27):3617-30

23. Khan SR. Reactive oxygen species as the molecular modulators of calcium oxalate kidney stone formation: evidence from clinical and experimental investigations. J Urol. 2013;189(3):803-11.

24. Verkoelen CF, Verhulst A. Proposed mechanisms in renal tubular crystal retention. Kidney Int. 2007;72(1):13-8.
25. Evan AP, Lingeman JE, Coe FL, Parks JH, Bledsoe SB, Shao Y, et al. Randall's plaque of patients with nephrolithiasis begins in basement membranes of thin loops of Henle. J Clin Investig. 2003;111(5):607-16.

26. Evan AP, Coe FL, Lingeman JE, Shao Y, Matlaga BR, Kim SC, et al. Renal crystal deposits and histopathology in patients with cystine stones. Kidney Int. 2006;69(12):2227-35.

27. Taylor ER, Stoller ML. Vascular theory of the formation of Randall plaques. Urolithiasis. 2015;43(1):41-5.

28. Pongsakul N, Vinaiphat A, Chanchaem P, Fong-Ngern K, Thongboonkerd V. Lamin $\mathrm{a} / \mathrm{C}$ in renal tubular cells is important for tissue repair, cell proliferation, and calcium oxalate crystal adhesion, and is associated with potential crystal receptors. FASEB J. 2016;30(10):3368-77.

29. Kusmartsev S, Dominguez-Gutierrez PR, Canales BK, Bird VG, Vieweg J, Khan SR, et al. Calcium oxalate stone fragment and crystal phagocytosis by human macrophages. J Urol. 2016;195(4):1143-51.

30. Zhao F, Qu Y, Zhu J, L Z, L H, Liu H, et al. miR-30d-5p plays an important role in autophagy and apoptosis in developing rat brains after hypoxicischemic injury. J Neuropathol Exp Neurol. 2017;76(8):709-19.

31. Duan $X$, Kong Z, Mai X, Lan Y, Liu Y, Yang Z, et al. Autophagy inhibition attenuates hyperoxaluria-induced renal tubular oxidative injury and calcium oxalate crystal depositions in the rat kidney. Redox Biol. 2018;16:414-25.

32. Zuo J, Khan A, Glenton PA, Khan SR. Effect of NADPH oxidase inhibition on the expression of kidney injury molecule and calcium oxalate crystal deposition in hydroxy-L-proline-induced hyperoxaluria in the male Sprague-Dawley rats. Nephrol Dial Transplant. 2011;26(6):1785-96.

33. Khan SR. Hyperoxaluria-induced oxidative stress and antioxidants for renal protection. Urol Res. 2005;33(5):349-57.

34. Umekawa T, Tsuji H, Uemura H, Khan SR. Superoxide from NADPH oxidase as second messenger for the expression of osteopontin and monocyte chemoattractant protein-1 in renal epithelial cells exposed to calcium oxalate crystals. BJU Int. 2009;104(1):115-20

35. Khan SR, Khan A, Byer KJ. Temporal changes in the expression of mRNA of NADPH oxidase subunits in renal epithelial cells exposed to oxalate or calcium oxalate crystals. Nephrol Dial Transplant. 2011;26(6):1778-85.

36. Gong Y, Hou J. Claudins in barrier and transport function-the kidney. Pflugers Arch - Eur J Physiol. 2017;469(1):105-13

37. Yu L, Gan X, Liu X, An R. Calcium oxalate crystals induces tight junction disruption in distal renal tubular epithelial cells by activating ROS/Akt/p38 MAPK signaling pathway. Ren Fail. 2017:39(1):440-51.

38. Wang XF, et al. Gastrin-releasing peptide receptor gene silencing inhibits the development of the epithelial-mesenchymal transition and formation of a calcium oxalate crystal in renal tubular epithelial cells in mice with kidney stones via the PI3K Akt signaling pathway. J Cell Physiol. 2019;234(2):1567-77.

39. Jeon SM, Chandel NS, Hay N. AMPK regulates NADPH homeostasis to promote tumour cell survival during energy stress. Nature. 2012;485(7400):661-5.

40. Tan SX, Teo M, Lam YT, Dawes IW, Perrone GG. Cu, Zn superoxide dismutase and $\mathrm{NADP}(\mathrm{H})$ homeostasis are required for tolerance of endoplasmic reticulum stress in Saccharomyces cerevisiae. Mol Biol Cell. 2009:20(5):1493-508.

41. Cao L, Chen J, Li M, Qin YY, Sun M, Sheng R, et al. Endogenous level of TIGAR in brain is associated with vulnerability of neurons to ischemic injury. Neurosci Bull. 2015;31(5):527-40.

42. Li M, Zhou ZP, Sun M, Cao L, Chen J, Qin YY, et al. Reduced nicotinamide adenine dinucleotide phosphate, a pentose phosphate pathway product, might be a novel drug candidate for ischemic stroke. Stroke. 2016:47(1):187-95.

43. Cochat P, Rumsby G. Primary hyperoxaluria. N Engl J Med. 2013;369(7):649-58.

44. Yu L, Zhang Y, Zhang H, Li Y. SOCS3 overexpression inhibits advanced glycation end product-induced EMT in proximal tubule epithelial cells. Exp Ther Med. 2017;13(6):3109-15.

45. Convento MB, Pessoa EA, Cruz E, da Glória MA, Schor N, Borges FT. Calcium oxalate crystals and oxalate induce an epithelial-to-mesenchymal transition in the proximal tubular epithelial cells: contribution to oxalate kidney injury. Sci Rep. 2017:7:45740.

46. Kanlaya R, Sintiprungrat K, Thongboonkerd V. Secreted products of macrophages exposed to calcium oxalate crystals induce epithelial mesenchymal transition of renal tubular cells via RhoA-dependent TGFbeta1 pathway. Cell Biochem Biophys. 2013:67(3):1207-15. 